\title{
Dificuldades percebidas na transição para a universidade
}

\section{Resumo}

\author{
Ana Cristina Garcia Dias ${ }^{1}$ \\ Universidade Federal do Rio Grande do Sul \\ Rodrigo Carvalho Carlotto \\ Instituto Federal Farroupilha \\ Clarissa Tochetto de Oliveira \\ Universidade Federal do Rio Grande do Sul \\ Marco Antônio Pereira Teixeira \\ Universidade Federal do Rio Grande do Sul
}

Este estudo investigou as principais dificuldades enfrentadas por estudantes universitários durante o ingresso no ensino superior e se a percepção das mesmas poderia variar em função do gênero, do semestre no curso, do fato do estudante estar frequentando o curso inicialmente desejado e tipo de instituição. Participaram dessa pesquisa 423 estudantes. Através de duas questões abertas, investigou-se as principais dificuldades acadêmicas e não acadêmicas vivenciadas pelos estudantes. As respostas foram submetidas a análise de conteúdo temática. A principal dificuldade acadêmica observada foi relacionada a diferenças entre o ensino médio e o superior. Já entre as dificuldades não acadêmicas, "gestão do tempo" foi a mais frequentemente citada. Alunos da Universidade tenderam a apresentar um pouco mais de dificuldades do que os do Instituto Federal. Implicações metodológicas e práticas dos resultados são discutidas.

Palavras-chave: eventos estressores; transição; estudantes universitários, dificuldades acadêmicas, dificuldades não acadêmicas.

\section{Abstract: Dificultades percibidas en la transición para la universidad}

This study investigated the main difficulties faced by undergraduate students when entering higher education, and whether their perception could vary according to gender, semester, if the student is attending the course initially desired and type of institution. 423 students participated in this study. Through two open questions, the main academic and non-academic difficulties experienced by the students were investigated. The answers were submitted to thematic content analysis. The main academic difficulty observed was related to differences between high school and higher education. Among the non-academic difficulties, "time management" was the most frequently cited. Students from the University tended to present a little more difficulties than those of the Federal Institute. Methodological and practical implications of the results were discussed.

Keywords: stressful events; transition; undergraduate students, academic difficulties, non-academic difficulties.

\section{Resumen: Dificuldades na transição para a universidade}

Este estudio investigó las principales dificultades enfrentadas por estudiantes universitarios al ingresar a la educación superior y si la percepción de las mismas podría variar con relación al género, semestre, hecho del estudiante estar frecuentando la carrera inicialmente deseada y tipo de institución. Participaron en la investigación 423 estudiantes. A través de dos cuestiones abiertas, se estudiaron las principales dificultades académicas y no académicas vivenciadas por los estudiantes. Las respuestas fueron sometidas a análisis de contenido temático. La principal dificultad académica observada está relacionada con las diferencias entre la enseñanza media y superior. Entre las dificultades no académicas, "la gestión del tiempo" fue citada con mayor frecuencia. Los estudiantes de la Universidad tienden a presentar un poco más de dificultades que los de la Institución Federal. Se discuten las implicaciones metodológicas y prácticas de los resultados.

Palabras clave: eventos estresantes; transición; estudiantes universitarios, dificultades académicas, dificultades no académicas.

\footnotetext{
1 Endereço para corresponência: Instituto de Psicologia, Rua Ramiro Barcelos, 2600 sala 207. Porto Alegre/RS CEP 90035-003
} E-mail: anacristinagarciadias@gmail.com 
O ingresso na universidade promove significativas mudanças na vida dos indivíduos. $\mathrm{O}$ estudante enfrenta uma série de desafios pessoais, interpessoais, familiares e institucionais (Oliveira \& Dias, 2014). Situações típicas desse período de transição como a saída de casa, alterações nas redes de amizade, exigências sociais de maior autonomia, dificuldades em lidar com a ausência de afeto, necessidade de constante aprovação dos pares, cobranças pelo bom desempenho acadêmico, problemas em administrar o tempo de forma adequada e outras demandas impostas pelo Ensino Superior (ES) podem se constituir em importantes estressores para universitários (Soares, Baldez, \& Mello, 2011).

De forma geral, entende-se por estressor todo e qualquer acontecimento, eventual ou sistemático, que desafie os limites e recursos psicológicos do sujeito (Lazarus \& Folkman, 1984). Quanto mais incontrolável ou imprevisível for um evento, maior sua probabilidade de ser percebido como estressante (Bardagi \& Hutz, 2011). Em geral, as fontes geradoras de estresse costumam ser organizadas em três categorias: macroestressores, microestressores e estados de tensão crônica. Macroestressores são eventos específicos que ocorrem ao longo da vida, podendo originar efeitos fisiológicos e psicológicos sob a forma de sintomas e desadaptação (ex. divórcios). Os micro-estressores referem-se às situações cotidianas que geram estresse a partir do acúmulo de diversos eventos de menor porte (ex. engarrafamento). Por sua vez, os estados de tensão crônica são caracterizados por experiências agressoras vividas através de anos, que podem causar patologias graves ao longo da vida (ex. violência infantil) (Margis, Picon, Cosner, \& Silveira, 2003).

$\mathrm{Na}$ literatura nacional e internacional, pesquisadores têm investigado os principais estressores enfrentados por estudantes durante a transição para o ES (Bardagi, 2007; Bondan \& Bardagi, 2008; García-Ros, Pérez-González, Pérez-Blasco, \& Natividad, 2012; Monteiro, Freitas, \& Ribeiro, 2007; Oliveira, Carlotto, Vasconcelos, \& Dias, 2014; Polo, Hernández, \& Pozo, 1996; Ross, Niebling, \& Heckert, 1999). Em boa parte dos casos, os estressores mais frequentes revelam discrepâncias entre as expectativas que o aluno tem e a realidade com a qual se depara no seu ingresso no ES. Nesse sentido, a decepção com os conteúdos das disciplinas, o desapontamento com professores e as dificuldades em relacionar a teoria com a prática profissional constituem-se em importantes fontes de estresse durante o primeiro ano (Bardagi, 2007). Questões relacionadas ao gerenciamento inadequado do tempo, a carga horária excessiva dos cursos, a realização de provas e as dificuldades enfrentadas em apresentar trabalhos em sala de aula também podem ser encarados como um problema para os estudantes (García-Ros et al., 2012). Em uma pesquisa com alunos de semestres iniciais de uma universidade privada do Rio Grande do Sul, situações relacionadas à escassez de tempo e à falta de motivação para estudar ou frequentar as aulas representavam importantes fontes de estresse entre alunos de cursos regulares de graduação. Já entre estudantes de cursos tecnológicos (mais voltados para demandas do mercado de trabalho e duração mais curta), a decepção com o conteúdo das disciplinas constituía-se no principal elemento estressor (Bondan \& Bardagi, 2008).

Algumas dificuldades também foram observadas em uma pesquisa realizada com calouros de Psicologia de uma universidade da Espanha. Foi apresentada aos estudantes uma lista com onze situações potencialmente geradoras de estresse acadêmico. Entre os estressores que receberam maior destaque estavam a falta de tempo para cumprir as atividades relacionadas à universidade, a desmotivação para estudar, a realização de provas, a exposição de trabalhos em classe, a má distribuição das disciplinas ao longo da semana e a sobrecarga acadêmica. Por outro lado, categorias como o trabalho em grupo e a competitividade entre colegas foram pouco mencionadas (Polo et al., 1996).

Outro estudo realizado com graduandos de diversos semestres de Enfermagem da Universidade Federal do Piauí, verificou que, em certos períodos da formação acadêmica, a distribuição inadequada de disciplinas repercutia de forma negativa na formação dos estudantes. $\mathrm{O}$ excesso de atividades acadêmicas associadas as dificuldades em organizar as atividades e reponsabilidades geravam altos níveis de tensão e estresse entre os universitários (Monteiro et al., 2007).

A percepção das dificuldades é influenciada por algumas variáveis. Questões de gênero e de estar ou não frequentando o curso inicialmente desejado podem interferir na forma como se percebe as dificuldades (Araújo et al. 2016; Christofoletti, Trelha, Galera, \& Feracin, 2007; Santos \& Alves Junior, 2007). Em termos de questões de gênero, as mulheres costumam apresentar maior prevalência de sintomas de estresse quando comparadas aos homens (Aguiar, Vieira, Vieira, Aguiar, \& Nóbrega, 2009; Calais et al., 2007; Chapell et al. 2005, Santos \& Alves Junior, 2007). Da mesma forma, estudantes que não frequentam o curso inicialmente desejado tendem apresentar níveis mais elevados de estresse e menores níveis de adaptação à universidade do que os que frequentam o curso desejado (Christofoletti et al., 2007; Oliveira \& Dias, 2013). Outros estudos indicam que o momento em que o estudante se encontra no curso também interfere nas vivências e expectativas relacionadas ao curso (Araújo \& Haas, 2012; Igue, Bariani \& Milanesi, 2008) ou aos níveis de estresse (Pereira, Miranda, \& Passos, 2010).

Em muitos casos, a presença de estressores ou dificuldades enfrentadas pode repercutir de maneira negativa em vários aspectos da vida do estudante, contribuindo para o desenvolvimento de comportamentos relacionados à depressão (Dahlin \& Runeson, 2007) e ao consumo de álcool (Phun \& Santos, 2010). Além disso, direta ou 
indiretamente, as situações estressoras podem contribuir para o insucesso acadêmico (Páramo et al., 2010), ou mesmo, abandono dos estudos (Bardagi, 2007).

A partir disso, considera-se necessária a realização de pesquisas que auxiliem na identificação de dificuldades envolvidas na transição para o ES. Uma vez mapeadas as principais dificuldades, pode-se, através de programas específicos, estimular o desenvolvimento de estratégias de enfrentamento que proporcionem ao estudante um manejo mais satisfatório das situações estressantes (Conley, Travers, \& Bryant, 2013; Horton, 2015; Misra \& Mckean 2000; Ramler, Tennison, Lynch, \& Murphy, 2016). Ademais, a identificação das principais fontes de preocupação e ansiedade no contexto universitário pode contribuir para o desenvolvimento de ações de combate à evasão acadêmica (Bardagi \& Hutz, 2011) e aumento da adaptação à universidade (Ko, 2015; Lee \& Eo, 2013). Nesse sentido, o presente estudo buscou conhecer as principais dificuldades acadêmicas e não acadêmicas enfrentadas por universitários durante o ES. Além disso, procurou-se verificar se as percepções das dificuldades vivenciadas no primeiro ano da graduação poderiam variar em função do gênero, do fato do aluno estar no ano inicial ou final do curso, do estudante estar ou não frequentando o curso inicialmente desejado e, em função do tipo de instituição [Universidade (Uni) ou Instituto Federal (IF)].

\section{Método}

\section{Participantes}

Participaram do estudo 423 estudantes (idade entre 16 a 47 anos, $M=23,3$ anos, $D P=5,27)$, de primeiro e último ano de uma universidade pública e de um Instituto Federal de Educação do interior do Rio Grande do Sul. A amostra contou com 274 mulheres $(64,8 \%)$ e 149 homens $(35,2 \%) ; 250(59,1 \%)$ estavam no primeiro ano e $173(40,9 \%)$ no ano final dos cursos: Medicina Veterinária (13,2\%), Educação Especial (12,1\%), Ciências Contábeis $(10,6 \%)$, Fonoaudiologia $(9,9 \%)$, Administração $(9,7 \%)$, Produção de Grãos (7,8\%), Enfermagem $(7,3 \%)$, Ciências Biológicas $(7,1 \%)$, Sistemas da Informação (6,4\%), Agronegócio (6,4\%), Matemática (5,0\%) e Artes Cênicas (4,5\%). Destes, 318 estudantes (75,2\%) estavam frequentando o curso inicialmente desejado (primeira opção).

Em função dos objetivos do estudo são apresentadas as duas instituições nas quais a pesquisa foi desenvolvida. A Uni é uma instituição pública de grande porte, com elevada procura nos 94 cursos de graduação ofertados. Localiza-se em uma cidade universitária de tamanho médio (278.445 habitantes). A cidade atrai estudantes de diversas regiões do estado e do país, que se mudam para cursar o ES na referida universidade ou em outras instituições da cidade. Já o IF situa-se em cidade pequena (20.012 habitantes), e oferece apenas seis cursos de nível superior.

\section{Instrumentos}

A fim de investigar as principais dificuldades vivenciadas pelos universitários durante o ES, foram realizadas duas perguntas abertas: "Ao entrar na universidade, você pode se deparar com dificuldades que não encontrava na sua escola. Você poderia descrever: a) Quais foram as dificuldades encontradas na universidade? b) Quais foram as dificuldades encontradas em outros contextos (casa, amigos etc.)?" Optou-se pela utilização do termo dificuldades, ao invés de estressores, pois o segundo pode apresentar uma conotação mais vinculada a psicopatologia do que o primeiro. Além dessas questões, os participantes responderam a outros instrumentos sobre vivências acadêmicas, estratégias de enfrentamento e autoeficácia profissional, que foram alvo de análises em outro estudo (Carlotto, Teixeira \& Dias, 2015).

\section{Procedimentos}

A pesquisa foi aprovada pelo Comitê de Ética em Pesquisa da Universidade Federal de Santa Maria (protocolo 12392413.4.0000.5346). O projeto foi apresentado às coordenações de cursos nas duas instituições de ensino. O questionário anônimo foi respondido coletivamente em sala de aula, sendo que nenhum jovem se recusou a participar.

\section{Análise das informações}

As informações obtidas foram submetidas à análise de conteúdo temática (Bardin, 2011). Inicialmente, três avaliadores realizaram uma leitura flutuante de todas as respostas, buscando identificar temas comuns. Após a discussão e definição das categorias temáticas as respostas foram analisadas e codificadas. Algumas respostas foram desmembradas, visto que apresentavam conteúdos distintos. As respostas foram quantificadas e tabuladas. Procedeu-se ao cálculo das frequências das categorias e no cômputo do número médio de dificuldades por subgrupo (homens e mulheres, calouros e formandos, cursando o curso desejado ou não, tipo de instituição). Os subgrupos foram comparados quanto à média de dificuldades relatadas através de testes de comparações de médias.

\section{Resultados}

Foram identificadas nove categorias de dificuldades (estressores) acadêmicas durante o ES. As categorias, 
com suas definições são: 1) Dificuldades interpessoais no ambiente acadêmico (problemas de relacionamento interpessoal que o estudante enfrentou em relação a colegas, professores e funcionários da instituição); 2) Diferenças entre o Ensino Médio (EM) e o ES (diz respeito às questões burocráticas, nível de exigência, autonomia e cobrança mais elevada presentes no ES); 3) Dificuldades econômicas (dificuldades financeiras enfrentadas no início da graduação); 4) Dificuldades de gestão do tempo relacionada às tarefas acadêmicas (problemas em conciliar as demandas da vida universitária); 5) Dificuldades cognitivas (problemas relacionados às capacidades de raciocínio, leitura, memória, processamento visual); 6) Dificuldades pessoais (características pessoais e situações particulares que geram estresse para cada estudante, por exemplo, timidez, insegurança, falta de motivação); 7) Dificuldades estruturais da universidade ou curso (dificuldades estruturais da instituição de ensino, tais como: biblioteca insuficiente ou defasada, falta de laboratórios adequados, etc.); 8) Dificuldades face a professores desinteressados ou sem habilidades didáticas adequadas (baixa habilidade ou desinteresse por alguns professores durante a condução das aulas); 9) Dificuldades de deslocamento e transporte (utilização mais frequente de meios de transporte, fato que pode representar um estressor para o estudante).

Já a segunda questão, na qual indagou-se sobre dificuldades (estressores) vivenciadas em outros contextos (casa, amigos etc.) foram identificadas seis categorias: 1) Dificuldades de gestão do tempo relacionada com a vida pessoal (o envolvimento intenso com atividades acadêmicas promove algumas dificuldades em relação à organização equilibrada do tempo. Muitas vezes, o estudante não dedica a atenção que julga necessária à sua família, amigos, cuidados de saúde e lazer); 2) Saudade da família (mudar de cidade para cursar o ES pode provocar saudade, tristeza); 3) Dificuldades de relacionamento com familiares e amigos (o acadêmico pode enfrentar dificuldades de relacionamento com familiares e amigos); 4) Falecimento ou doença de familiares ou amigos (doença ou perda de entes queridos); 5) Pouco apoio da família e/ou amigos (família e amigos não apoiam emocionalmente a decisão de cursar o ES); e 6) Outros (respostas não compatíveis aos objetivos da pergunta, ou que não apresentaram frequência que justificasse a criação de uma categoria própria).

Na Tabela 1 são apresentadas as principais dificuldades acadêmicas relatadas associadas à transição para o ensino superior.

$\mathrm{Na}$ Tabela 2, são descritas as principais dificuldades acadêmicas relatadas por homens e mulheres, calouros e formandos, alunos que frequentam (ou não) o curso inicialmente desejado.

As categorias com maior percentual de respostas foram "Diferenças entre o EM e o ES", "Não percebeu dificuldades", exceto no contraste entre os estudantes da Uni e do IF, onde se verifica que os estudantes do IF tenderam a perceber menos dificuldades do que os da Uni. Testes de associação realizados (utilizando o método exato de Fisher) indicaram diferenças estatisticamente significativas na comparação entre os alunos da Uni e do IF nas categorias "Não percebeu dificuldades" $(p=0,029)$, "Diferenças entre o $\mathrm{EM}$ e o $\mathrm{ES}$ " $(p=0,036)$ e "Interpessoais" $(p=0,045)$. Nas comparações entre os demais grupos não foram observadas diferenças estatisticamente significativas nos percentuais de cada categoria $(p s>0,05)$.

$\mathrm{Na}$ Tabela 3 são apresentadas as principais dificuldades (estressores) encontradas em contextos não acadêmicos (como casa, amigos etc.) durante a transição para o ES.

Tabela 1

Frequências e Porcentagens para cada Dificuldade Acadêmica

\begin{tabular}{lcc}
\hline Tipo de dificuldade acadêmica relatada & Frequência & Percentual \\
\hline Diferenças entre o EM e o ES & 120 & 29,7 \\
Pessoais & 80 & 18,9 \\
Interpessoais & 70 & 17,3 \\
Gestão do tempo relacionada às tarefas acadêmicas & 65 & 16,1 \\
Estrutura da universidade ou do curso & 37 & 9,2 \\
Cognitivas & 26 & 6,4 \\
Professores desinteressados ou sem habilidades didáticas adequadas & 19 & 4,7 \\
Deslocamento e transporte & 18 & 4,5 \\
Econômicas & 16 & 4,0 \\
Não percebeu dificuldades & 91 & 22,5 \\
\hline
\end{tabular}

Nota: As frequências indicam o número de participantes cujas respostas foram enquadradas nas categorias descritas, e o percentual, para cada categoria, foi calculado em relação ao total da amostra que respondeu a esta questão ( $n=404$; 19 não responderam a questão). 
Tabela 2

Frequências e Porcentagens (entre parênteses) para cada Dificuldade Acadêmica em Grupos Distintos

\begin{tabular}{lcccccccc}
\hline $\begin{array}{l}\text { Tipo de dificuldade } \\
\text { acadêmica relatada }\end{array}$ & \multicolumn{2}{c}{ Sexo } & \multicolumn{2}{c}{ Etapa do curso } & \multicolumn{2}{c}{ Curso desejado } & \multicolumn{2}{c}{ Instituição } \\
\hline & Fem. & Mas. & Início & Fim & Sim & Não & Uni. & IF \\
& $(n=260)$ & $(n=144)$ & $(n=240)$ & $(n=164)$ & $(n=303)$ & $(n=96)$ & $(n=237)$ & $(n=167)$ \\
Diferenças entre EM e ES & 81 & 39 & 67 & 53 & 92 & 26 & 80 & 40 \\
& $(31,2)$ & $(27,1)$ & $(27,9)$ & $(32,3)$ & $(30,4)$ & $(27,1)$ & $(33,8)$ & $(20,4)$ \\
Pessoais & 52 & 28 & 50 & 30 & 60 & 20 & 53 & 27 \\
& $(20,0)$ & $(19,4)$ & $(20,8)$ & $(18,3)$ & $(19,8)$ & $(20,8)$ & $(22,4)$ & $(16,2)$ \\
Interpessoais & 48 & 22 & 39 & 31 & 53 & 15 & 49 & 21 \\
& $(18,5)$ & $(15,3)$ & $(16,3)$ & $(18,9)$ & $(17,5)$ & $(15,6)$ & $(20,7)$ & $(12,6)$ \\
Gestão do tempo b & 41 & 24 & 39 & 26 & 46 & 18 & 35 & 30 \\
& $(15,8)$ & $(16,7)$ & $(16,3)$ & $(15,9)$ & $(15,2)$ & $(18,8)$ & $(14,8)$ & $(18,0)$ \\
Estruturac & 26 & 11 & 22 & 15 & 31 & 5 & 25 & 12 \\
& $(10,0)$ & $(7,6)$ & $(9,2)$ & $(9,1)$ & $(10,2)$ & $(5,2)$ & $(10,5)$ & $(7,2)$ \\
Cognitivas & 18 & 8 & 13 & 13 & 21 & 5 & 16 & 10 \\
& $(6,9)$ & $(5,6)$ & $(5,4)$ & $(7,9)$ & $(6,9)$ & $(5,2)$ & $(6,8)$ & $(6,0)$ \\
Professores & 11 & 8 & 8 & 11 & 12 & 7 & 13 & 6 \\
& $(4,2)$ & $(5,6)$ & $(3,3)$ & $(6,7)$ & $(4,0)$ & $(7,3)$ & $(5,5)$ & $(3,6)$ \\
Deslocamento e transporte & 15 & 3 & 11 & 7 & 13 & 4 & 11 & 7 \\
& $(5,8)$ & $(2,1)$ & $(4,6)$ & $(4,3)$ & $(4,3)$ & $(4,2)$ & $(4,6)$ & $(4,2)$ \\
Econômicas & 8 & 8 & 9 & 7 & 10 & 6 & 8 & 8 \\
& $(3,1)$ & $(5,6)$ & $(3,8)$ & $(4,3)$ & $(3,3)$ & $(6,3)$ & $(3,4)$ & $(4,8)$ \\
Não percebeu dificuldades & 56 & 35 & 57 & 34 & 66 & 24 & 44 & 47 \\
& $(21,5)$ & $(24,3)$ & $(23,8)$ & $(20,7)$ & $(21,8)$ & $(25,0)$ & $(18,6)$ & $(28,1)$ \\
\hline
\end{tabular}

Nota: As frequências indicam o número de participantes cujas respostas foram enquadradas nas categorias descritas, e o percentual, para cada categoria, foi calculado em relação ao $n$ de cada subgrupo. Cinco participantes não informaram se o curso que frequentavam era o desejado e não foram incluídos na análise relativa a esta variável.

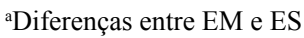

${ }^{\mathrm{b}}$ Gestão do tempo relacionada às tarefas acadêmicas

${ }^{c}$ Estrutura da universidade ou do curso

dProfessores desinteressados ou sem habilidades didáticas adequadas

$\mathrm{Na}$ Tabela 4, apresentam-se as principais dificuldades encontradas em contextos não acadêmicos relatadas pelos grupos.

Verifica-se que em todos os grupos analisados, as categorias: "Não percebeu dificuldades" e "Gestão do tempo" foram as que apresentaram frequências mais altas. Testes de associação realizados (utilizando o método exato de Fisher) indicaram diferenças estatisticamente significativas na comparação entre os alunos da Uni e do IF nas categorias "Não percebeu dificuldades" $(p=0,008)$, "Saudade da família" $(p<0,001)$. Nas comparações entre os demais grupos não foram observadas diferenças estatisticamente significativas nos percentuais de cada categoria $(p \mathrm{~s}>0,05)$.

Como análise complementar, e com o intuito de possibilitar uma comparação entre os grupos em termos do número de dificuldades relatadas, foram computados os somatórios de dificuldades encontradas em contextos acadêmicos e não acadêmicos. Assim, o escore de cada participante podia se situar entre 0 e 9 (dificuldades acadêmicas) e 0 e 6 (dificuldades não acadêmicas). As médias dos grupos foram comparadas através de testes $t$. A Tabela 5 traz os resultados dessas comparações. Apenas na comparação entre Uni e IF foram observadas diferenças estatisticamente significativas, sendo que mais dificuldade foram percebidas na Uni. Três análises adicionais foram ainda realizadas: comparando homens e mulheres considerando o grupo de formandos e de calouros separadamente; comparando calouros e formandos considerando o grupo de homens e mulheres separadamente; e comparando estudantes em curso desejado e não desejado considerando o grupo de calouros e formandos 
Tabela 3

Frequências e Porcentagens para cada Dificuldade Encontrada em Contexto Não Acadêmico

\begin{tabular}{lcc}
\hline Tipo de dificuldade não acadêmica relatada & Frequência & Percentual \\
\hline Gestão do tempo & 89 & 25,6 \\
Saudade da família & 53 & 15,3 \\
Relacionamento com familiares e amigos & 40 & 11,5 \\
Pouco apoio da família e/ou amigos & 10 & 2,9 \\
Falecimento ou doença de familiares ou amigos & 12 & 3,5 \\
Outros & 13 & 3,7 \\
Não percebeu dificuldades & 142 & 40,9 \\
\hline
\end{tabular}

Nota: As frequências indicam o número de participantes cujas respostas foram enquadradas nas categorias descritas, e o percentual, para cada categoria, foi calculado em relação ao total da amostra que respondeu a esta questão ( $\mathrm{n}=347 ; 76$ não responderam à questão).

Tabela 4

Frequências e Porcentagens (entre parênteses) para cada Dificuldade Não Acadêmica em Grupos Distintos

\begin{tabular}{lcccccccc}
\hline \multirow{2}{*}{$\begin{array}{l}\text { Tipo de dificuldade } \\
\text { acadêmica relatada }\end{array}$} & \multicolumn{2}{c}{ Sexo } & \multicolumn{2}{c}{ Etapa do curso } & \multicolumn{2}{c}{ Curso desejado } & \multicolumn{2}{c}{ Instituição } \\
\cline { 2 - 8 } & Fem. & Mas. & Início & Fim & Sim & Não & Uni. & IF \\
$(n=260)$ & $(n=144)$ & $(n=240)$ & $(n=164)$ & $(n=303)$ & $(n=96)$ & $(n=201)$ & $(n=146)$ \\
\hline Gestão do tempo & 57 & 32 & 58 & 31 & 68 & 20 & 49 & 40 \\
& $(24,6)$ & $(27,8)$ & $(27,5)$ & $(22,8)$ & $(26,2)$ & $(23,8)$ & $(24,4)$ & $(27,4)$ \\
Saudade da família & 39 & 14 & 36 & 17 & 39 & 13 & 43 & 10 \\
& $(16,8)$ & $(12,2)$ & $(17,1)$ & $(12,5)$ & $(15,0)$ & $(15,5)$ & $(21,4)$ & $(6,8)$ \\
Relacionamento com & 27 & 13 & 20 & 20 & 32 & 8 & 27 & 13 \\
familiares e amigos & $(11,6)$ & $(11,3)$ & $(9,5)$ & $(14,7)$ & $(12,3)$ & $(9,5)$ & $(13,4)$ & $(8,9)$ \\
Pouco apoio da família & 8 & 2 & 7 & 3 & 7 & 3 & 3 & 7 \\
e/ou amigos & $(3,4)$ & $(1,7)$ & $(3,3)$ & $(2,2)$ & $(2,7)$ & $(3,6)$ & $(1,5)$ & $(4,8)$ \\
Falecimento / doença & 11 & 1 & 7 & 5 & 9 & 3 & 9 & 3 \\
& $(4,7)$ & $(0,9)$ & $(3,3)$ & $(3,7)$ & $(3,5)$ & $(3,6)$ & $(4,5)$ & $(2,1)$ \\
Outros & 7 & 6 & 8 & 5 & 9 & 4 & 8 & 5 \\
& $(3,0)$ & $(5,2)$ & $(3,8)$ & $(3,7)$ & $(3,5)$ & $(4,8)$ & $(4,0)$ & $(3,4)$ \\
Não percebeu & 92 & 50 & 83 & 59 & 104 & 37 & 70 & 72 \\
dificuldades & $(39,7)$ & $(43,5)$ & $(39,3)$ & $(43,4)$ & $(40,0)$ & $(44)$ & $(34,8)$ & $(49,3)$ \\
\hline
\end{tabular}

Nota: As frequências indicam o número de participantes cujas respostas foram enquadradas nas categorias descritas, e o percentual, para cada categoria, foi calculado em relação ao $n$ de cada subgrupo. Três participantes não informaram se o curso que frequentavam era o desejado e não foram incluídos na análise relativa a esta variável.

aFalecimento ou doença de familiares ou amigos

separadamente. Em nenhuma destas comparações foi registrada diferença estatisticamente significativa.

\section{Discussão}

O objetivo deste estudo foi descrever as principais dificuldades enfrentadas por estudantes universitários durante o ES. Além disso, procurou-se verificar se as percepções das dificuldades vivenciadas na graduação poderiam variar em função do gênero, do fato do aluno estar no ano inicial ou final de seu curso, do fato do acadêmico estar frequentando o curso que inicialmente desejou ou não, e também em função do tipo de instituição (Uni ou IF). Através das análises de frequência foram examinadas as principais dificuldades acadêmicas e não acadêmicas mencionadas pelos estudantes. Posteriormente foram analisadas as médias para totais de dificuldades relatadas pelos grupos.

De maneira geral, as dificuldades mais recorrentes relatadas pelos participantes do estudo convergem com os achados de outras pesquisas desenvolvidas junto ao público universitário (Costa \& Polak, 2009; Ross et al., 1999). Tem-se observado que a maior parte 
Tabela 5

Comparações de médias para totais de dificuldades relatadas

\begin{tabular}{|c|c|c|c|c|c|c|c|c|c|c|}
\hline & \multicolumn{5}{|c|}{ Dificuldades acadêmicas } & \multicolumn{5}{|c|}{ Dificuldades não acadêmicas } \\
\hline & $n$ & $M$ & $D P$ & $T$ & $p$ & $n$ & $M$ & $D P$ & $t$ & $p$ \\
\hline Sexo & & & & 1,16 & 0,247 & & & & 0,80 & 0,423 \\
\hline Fem & 260 & 1,15 & 0,87 & & & 232 & 0,64 & 0,56 & & \\
\hline Mas & 144 & 1,05 & 0,88 & & & 115 & 0,59 & 0,54 & & \\
\hline Etapa do curso & & & & 1,15 & 0,250 & & & & 0,80 & 0,425 \\
\hline Calouro & 240 & 1,08 & 0,87 & & & 211 & 0,64 & 0,55 & & \\
\hline Formando & 164 & 1,18 & 0,89 & & & 136 & 0,60 & 0,56 & & \\
\hline Curso desejado & & & & 0,11 & 0,912 & & & & 0,33 & 0,736 \\
\hline $\operatorname{Sim}$ & 303 & 1,12 & 0,84 & & & 260 & 0,63 & 0,55 & & \\
\hline Não & 96 & 1,10 & 0,96 & & & 84 & 0,61 & 0,58 & & \\
\hline Instituição & & & & 2,97 & 0,003 & & & & 2,68 & 0,008 \\
\hline Uni & 237 & 1,22 & 0,90 & & & 201 & 0,70 & 0,55 & & \\
\hline IF & 167 & 0,96 & 0,81 & & & 146 & 0,53 & 0,57 & & \\
\hline
\end{tabular}

dos estressores vivenciados durante a transição para o ES está relacionada a aspectos pessoais, interpessoais, familiares e institucionais (Costa \& Polak, 2009). No entanto, chama a atenção o fato de um número considerável de alunos não ter indicado percepção de dificuldades durante o seu ingresso na universidade. Na medida em que o primeiro ano da graduação é caracterizado por um número significativo de estressores (Guerreiro-Casanova \& Polydoro, 2010), não se esperava que a não percepção de dificuldades fosse tão prevalente na amostra estudada. Provavelmente, aspectos ligados à euforia e à idealização em relação ao novo ambiente educacional possam ter colaborado para esses resultados (Polydoro, 2000). Outra possibilidade para essa divergência de achados é a metodologia de investigação utilizada, na qual se solicitou a evocação espontânea de dificuldades, ao invés de oferecer uma lista de dificuldades/estressores previamente descritos os quais devem ser assinalados pelos participantes. Outra possível explicação para essa menor frequência de percepção de dificuldades pode ser o fato de que as questões analisadas neste artigo fizeram parte de um estudo maior que buscou conhecer as estratégias de enfrentamento que os estudantes utilizavam para se adaptar à universidade. Nesse sentido, pode ser que, ao perceberem os próprios recursos de enfrentamento, os participantes tenham minimizado ou não percebido como relevantes algumas das dificuldades indicadas em outros estudos.

Entre as dificuldades acadêmicas mencionadas, a categoria "Diferenças entre o ensino médio e o superior" foi a mais relevante. Os principais problemas decorrem do processo adaptativo e estão relacionados a níveis de exigência pessoal e acadêmica a que o estudante não estava acostumado no ensino médio (Cunha \& Carrilho, 2005). A cobrança mais elevada por parte dos professores, a necessidade de um nível mais alto de autonomia e a presença de uma instituição mais complexa em termos de regras e burocracias pode ter repercussões negativas na vida dos acadêmicos. Muitas vezes, essas mudanças acabam gerando um aumento da responsabilidade, ansiedade, competição e de incertezas em relação à escolha profissional, questões essas que, se não forem bem conduzidas, podem representar importantes estressores para o estudante (Calais et al., 2007).

Outras duas importantes fontes de estresse descritas pelos estudantes, relacionadas ao contexto acadêmico, foram as categorias "Dificuldades pessoais" e "Dificuldades Interpessoais". Verifica-se que aspectos pessoais relacionados à insegurança e à desmotivação costumam representar importantes fontes de estresse para os jovens (Cardoso \& Bzuneck, 2004). Da mesma forma, dificuldades de relacionamento com colegas e professores também podem se constituir em estressores significativos para os acadêmicos. Se, por um lado, a diversidade das relações interpessoais estabelecidas no ambiente universitário pode favorecer o crescimento pessoal, por outro as características diferentes desses relacionamentos, comparados aos relacionamentos vivenciados durante o EM, podem também ser fonte de estresse. A dificuldade de relação entre os pares pode gerar rivalidades, falta de apoio e atribuição de culpa por erros ou problemas, podendo em alguns casos conduzir o aluno ao isolamento social. Muitas vezes, esses fatores contribuem para o surgimento de tensões psicológicas e sentimentos de insatisfação em relação ao ambiente acadêmico (Sadir \& Lipp, 2009). Além disso, cabe lembrar que estudantes que apresentam problemas 
em seus contatos sociais costumam ser mais propensos ao desenvolvimento de quadros ansiosos (Cruz, 2008).

De forma não menos importante, cabe ainda destacar a categoria "Dificuldades de gestão do tempo relacionada às tarefas acadêmicas". O tempo é um recurso limitado para os graduandos. Uma rotina elevada de leituras e avaliações, prazos mais rígidos para entrega de trabalhos, e um maior número de atividades extracurriculares são elementos que costumam interferir na organização do tempo dos estudantes (MacCann, Fogarty, \& Roberts, 2012). Diante disso, não é raro que a dificuldade em administrar compromissos possa provocar no acadêmico uma menor percepção de controle sobre aspectos pessoais e profissionais de sua vida, consequentemente tornando-o uma pessoa mais estressada e insatisfeita (Leite, Tamoyo, \& Gunter, 2003).

Já em relação às dificuldades vivenciadas em contextos não acadêmicos (casa, amigos etc.), verificou-se que os principais estressores mencionados estavam relacionados às categorias "Dificuldades relacionadas à gestão do tempo" e "Saudade da família", embora a categoria mais frequente tenha sido "Não percebeu dificuldades". Sabe-se que o ingresso na universidade é marcado por uma série de desafios pessoais, interpessoais, familiares e institucionais (Bondan \& Bardagi, 2008). Surpreende o fato de um número significativo de estudantes não terem percebido dificuldades não acadêmicas durante esse período. Talvez alguns ganhos ou benefícios proporcionados pelo ingresso no ensino superior, tais como o sentimento de liberdade e autonomia, a aquisição de novas experiências e o desenvolvimento de habilidades sociais possam ganhar maior relevância na percepção dos estudantes, fazendo com que as dificuldades sejam minimizadas (Calais et al., 2007).

Apesar da frequência não tão elevada, as categorias "Dificuldades de gestão do tempo" e "Saudade da família" agrupam importantes estressores não acadêmicos vivenciados pelos estudantes. Em muitos casos, devido ao envolvimento com as novas tarefas acadêmicas, o universitário destina pouco tempo às atividades de lazer e convivência com familiares e amigos (Pereira, Miranda, $\&$ Passos, 2010). Esses autores indicam que as demandas relacionadas à saúde (poucas horas de sono, alimentação inadequada, falta de lazer), à casa/família (tarefas domésticas), ao trabalho / universidade (avaliações, sobrecarga e atividades extracurriculares), além de preocupações financeiras podem gerar alterações psicológicas importantes no indivíduo. $\mathrm{O}$ estudante que não distribui de forma equilibrada seus horários e gerencia de forma equivocada seus compromissos estará mais propenso a desenvolver sintomas relacionados ao estresse, ansiedade e depressão (Soares, 2008). Além disso, Pereira et al. (2010) observam que os próprios estressores presentes no contexto universitário podem levar o indivíduo ao isolamento. Este, ao se afastar do convívio familiar e social, pode perceber-se sem apoio e só, o que, por sua vez, agrava os níveis de estresse vividos.

Nesse sentido, tem-se constatado que morar longe da família pode representar um importante estressor para o estudante. Alguns estudos têm verificado que os discentes que saem da casa dos pais para frequentar uma universidade costumam manifestar maiores dificuldades no processo de adaptação acadêmica (Scopelliti \& Tiberio, 2010). Questões relacionadas ao retraimento social e à falta de habilidade para lidar com a autonomia proporcionada por esse novo momento de vida podem explicar, em parte, as dificuldades de alguns alunos em morar longe dos pais (Guerra, Lencastre, Lemos, \& Pereira, 2002).

Cabe ressaltar, dentre os resultados, que os estressores relacionados à gestão inadequada do tempo e dificuldade de relacionamento interpessoal fizeram-se presentes nos contextos acadêmico e não acadêmico. Independentemente do ambiente ou público avaliado, observa-se que os problemas relacionados à administração do tempo constituem-se em importantes estressores para os sujeitos durante a universidade (Claessens, Eerde, Rutte, \& Roe, 2007; Pimienta, Cruz \&, Diaz-Véliz, 2016) e, portanto, podem ser alvo de intervenção junto a essa população (Oliveira, Carlotto, Teixeira, \& Dias, 2016). Da mesma forma, é compreensível que dificuldades ligadas aos relacionamentos interpessoais possam representar fontes de estresse em ambos os contextos. Normalmente, o ingresso na universidade é marcado por significativas alterações nas redes de amizade e nos laços familiares, fato que aumenta a probabilidade de contatos sociais insatisfatórios (Santos, Oliveira, \& Dias, 2015; Scopelliti \& Tiberio, 2010).

As análises de frequência e médias mostraram que a percepção dos estressores, tanto no contexto acadêmico quanto no não acadêmico, não variou em função do gênero, etapa do curso e do fato do curso frequentado ser desejado ou não. Foram observadas apenas algumas diferenças entre os estudantes da universidade e do Instituto Federal.

A ausência de diferenças entre homens e mulheres chama a atenção, pois há evidências de que as mulheres percebem ou antecipam mais estressores acadêmicos do que os homens (Aguiar et al., 2009; Araújo et al., 2016; Garcia-Ros, Pérez-González, Pérez-Blasco, \& Natividad, 2012; Madhyastha, Latha, \& Kamath, 2014; Santos \& Alves Junior, 2007). Já em relação à etapa do curso, embora não tenham sido localizadas pesquisas que indicassem possíveis diferenças entre calouros e formandos, imaginava-se que os tipos de estressores percebidos por estes dois grupos poderiam ser diferentes, ou mesmo que os calouros percebessem mais estressores, uma vez que estariam se adaptando a um novo ambiente e a novas demandas. Da mesma maneira, seria de esperar que os alunos que não estivessem no curso 
inicialmente desejado pudessem perceber um número maior de dificuldades durante o seu ingresso na universidade. Imaginava-se que esse grupo de alunos estivesse mais predisposto a apresentar comportamentos associados à desmotivação e à falta de engajamento em relação ao curso e à carreira, sendo, consequentemente, mais vulnerável aos estressores (Christofoletti et al., 2007). Uma explicação provável para a ausência de diferenças observada nesta pesquisa é o método usado para avaliar a percepção de estressores, como já indicado anteriormente. Uma vez que a avaliação dos estressores foi baseada apenas na presença ou não das dificuldades, a partir do relato espontâneo dos participantes, é possível que estressores menos evidentes no cotidiano sequer tenham sido lembrados pelos estudantes. Além disso, não foi avaliada também a intensidade do incômodo ou desajustamento causado pelos estressores. Dessa forma, o método utilizado pode não ter sido sensível o suficiente para captar a variabilidade existente entre os participantes no que diz respeito às dificuldades experimentadas na vida acadêmica ou em decorrência dela.

As diferenças verificadas entre Uni e IF ensejam algumas considerações. Embora não houvesse expectativas a priori para possíveis diferenças entre os grupos, os resultados sugerem que as características do contexto podem apresentar relação com as dificuldades percebidas pelos estudantes. De um modo geral, os estudantes da Uni perceberam mais dificuldades do que os do IF, especialmente aquelas relacionadas com as diferenças entre o ensino médio e superior, as relações interpessoais e a saudade de casa. Uma vez que a Uni em questão, em comparação com o IF, é uma instituição muito maior, com mais cursos e mais alunos, é também possivelmente um ambiente mais impessoal e menos acolhedor ou sensível às necessidades dos alunos, o que pode gerar mais dificuldades de adaptação à vida no ensino superior. A sensação de ser apenas mais um aluno dentro de uma instituição com milhares de outros alunos pode amplificar a percepção sobre as diferenças entre ensino médio e superior, assim como dificultar o estabelecimento de relações interpessoais. Por fim, o fato da Uni estar localizada em uma cidade universitária, para a qual muitos estudantes se mudam com o intuito de estudar, também pode explicar as diferenças observadas nos sentimentos de saudade da família.

\section{Considerações finais}

Esta pesquisa buscou mapear possíveis dificuldades vivenciadas pelos universitários durante o ingresso no ensino superior. Os tipos de dificuldades identificadas se assemelharam ao que é relatado na literatura, embora certas diferenças descritas em outros estudos não tenham sido observadas. Isso chama a atenção para um importante fator metodológico a ser considerado nas pesquisas sobre percepção de estressores e outras vivências acadêmicas: o fato da avaliação ser baseada em respostas não induzidas (espontâneas) ou induzidas por itens de escalas ou questionários. Os resultados deste estudo fazem pensar que as dificuldades vivenciadas no ensino superior talvez nem sempre sejam percebidas ou nominadas como dificuldades, embora possam ser experimentadas no dia a dia.

Os achados deste estudo precisam também ser considerados dentro de seu contexto e limitações. Participaram desta pesquisa estudantes de apenas duas instituições de ensino superior do interior de um único estado do país. Assim, qualquer generalização dos resultados deve ser feita com cautela, pois fatores específicos da amostra, como as características das instituições e mesmo das cidades em que estão situadas pode ter relação com a percepção de estressores decorrentes da vida acadêmica.

Apesar das limitações, cabe ressaltar algumas possíveis implicações para a teoria sobre o ajustamento à universidade e a prática do aconselhamento com populações universitárias. Em termos teóricos, verifica-se a necessidade de distinguir conceitualmente dificuldades ou estressores manifestos ou expressos (ou seja, aquelas relatadas espontaneamente) daquelas dificuldades inventariadas (induzidas por instrumentos), uma vez que isso pode conduzir a diferentes tipos de resultados. Essa distinção tem também implicações práticas para quem orienta estudantes no ensino superior, pois sugere a necessidade de uso de instrumentos mais objetivos que oferecem um repertório de possibilidades que estimulem a reflexão do estudante sobre a sua experiência na universidade. A ausência de instrumentos desse tipo pode levar a uma subestimação das dificuldades vivenciadas pelos alunos e com isso restringir o alcance de ações de suporte voltadas a essa população.

Uma direção para estudos futuros é investigar quais características cognitivas, motivacionais e comportamentais desses alunos podem ter contribuído para os elevados índices observados na categoria "Não percebeu dificuldades". Além disso, acredita-se que o desenvolvimento de pesquisas que tanto analisem a intensidade e o desconforto provocado pelos principais estressores, assim como outras variáveis (exemplo: nível socioeconômico, apoio familiar, raça) também podem ser úteis para compreensão dos estressores acadêmicos e não acadêmicos. Outra linha de investigação pode explorar ainda os estressores vivenciados especificamente em cada curso pesquisado, algo que não foi possível analisar neste estudo pelo fato de não haver uma quantidade proporcional e suficientemente grande de participantes por curso. Apesar das limitações apresentadas, acredita-se que os achados desse estudo contribuem para um melhor entendimento dos estressores vivenciados pelos acadêmicos, proporcionando um panorama ampliado sobre esse fenômeno. 


\section{Referências}

A Aguiar, S. M., Vieira, A. P. G. F., Vieira, K. M. F., Aguiar, S. M., \& Nóbrega, J. O. (2009). Prevalência de sintomas de estresse nos estudantes de medicina. Jornal Brasileiro de Psiquiatria, 58(1), 34-38. doi:10.1590/ S0047-20852009000100005

Araújo, A. M., et al. (2016). Dificuldades antecipadas de adaptação ao ensino superior: um estudo com alunos do primeiro ano. Revista de Estudios e Investigación em Psicología y Educación, 3(2), 102-111. doi:10.17979/reipe.2016.3.2.1846

Araújo, R. F., \& Haas, C. M. (2012). Alunos ingressantes e concluintes de curso universitário: perfis, expectativas e satisfação.Revista@mbienteeducação, 5(1),110-125.

Bardagi, M. P. (2007). Evasão e comportamento vocacional de universitários. Estudos sobre o desenvolvimento de carreira na graduação. (Tese de Doutorado, Programa de Pós-Graduação em Psicologia da Universidade Federal do Rio Grande do Sul).

Bardagi, M. P., \& Hutz, C. S. (2011). Eventos estressores no contexto acadêmico: uma breve revisão da literatura. Interação em Psicologia, 15(1), 111-119. doi:10.5380/psi.v151.1.17085

Bardin, L. (2011). Análise de Conteúdo. Lisboa: Edições 70.

Bondan, A. P., \& Bardagi, M. P. (2008). Comprometimento profissional e estressores percebidos por graduandos regulares e tecnológicos. Paideia, 18(41), 581-590. doi:10.1590/S0103-863X2008000300013.

Calais, S. L., Carrara, K., Brum, M. M., Batista, K., Yamada, J. K., \& Oliveira, J. R. S. (2007). Stress entre calouros e veteranos de jornalismo. Estudos de Psicologia (Campinas), 24(1), 69-77. doi:10.1590/S0103-166X2007000100008.-

Cardoso, L., \& Bzuneck, J. (2004). Motivação no ensino superior: metas de realização e estratégias de aprendizagem. Psicologia Escolar e Educacional, 8, 145-155.

Carlotto, R. C., Teixeira, M.A.P., Dias, A.C.G. (2015). Adaptação Acadêmica e Coping em Estudantes Universitários. Psico-USF, 20 (3), 421-432.

Chapell, M. S., et al. (2005). Test anxiety and academic performance in undergraduate and graduate students. Journal of Educational Psychology, 97(2), 268-274. doi:10.1037/0022-0663.97.2.268

Christofoletti G, Trelha C. S., Galera R. M., \& Feracin M. A. (2007). Síndrome de burnout em acadêmicos de fisioterapia. Fisioterapia e Pesquisa, 14(2), 35-39. doi:10.1590/fpusp.v14i2.75857

Claessens, B. J. C., Van Eerde, W., Rutte, C. G., \& Roe, R. A. (2007). A review of the time management literature. Personnel Review, 36(2), 255-276. doi:10.1108/00483480710726136

Clinciu, A. I. (2013). Adaptation and stress for the first year university students. Procedia-Social and Behavioral Sciences, 78, 718-722. doi:10.1016/j.sbspro.2013.04.382

Conley, C. S., Travers, L. V., \& Bryant, F. B. (2013). Promoting psychosocial adjustment and stress management in firstyear college students: The benefits of engagement in a psychosocial wellness seminar. Journal of American College Health, 61(2), 75-86. doi:10.1080/07448481.2012.754757

Costa, A. L. S., \& Polak, C. (2009). Construção e validação de instrumento para avaliação de estresse em estudantes de enfermagem (AEEE) [Número especial]. Revista da Escola de Enfermagem da USP, 43(Esp.), 1017-1026. doi:10.1590/S0080-62342009000500005

Cruz, M. A. C. (2008). Ansiedade e bem-estar na transição para o Ensino superior: o papel do suporte social. (Dissertação de Mestrado, Programa de Pós-Graduação em Psicologia e de Ciências da Educação da Universidade do Porto).

Cunha, S. M., \& Carrilho, D. M. (2005). O processo de adaptação ao ensino superior e o rendimento acadêmico. Psicologia Escolar e Educacional, 9(2), 215-224. doi:10.1590/S1413-85572005000200004

Dahlin, M., \& Runeson, B. (2007). Burnout and psychiatric morbidity among medical students entering clinical training: a three year prospective questionnaire and interview-based study. BMC Medical Education, 7:6. doi:10.1186/1472-6920-7-6

Freire, H. B. G., \& Noriega, J. A. V. (2011). Coping em Estudantes Universitários: Relação com Áreas do Conhecimento. Psicologia para América Latina, 21, 2-14. 
García-Ros, R., Pérez-González, F., Pérez-Blasco, J., \& Natividad, L. A. (2012). Evaluación del estrés académico em estudiantes de nueva incorporación a la universidad. Revista Latinoamericana de Psicología, 44(2), 143- 154.

Guerra, M. P., Lencastre, L., Lemos, M. S., \& Pereira, D. C. (2002). Problemas psicossociais dos estudantes do $1 .^{\circ}$ ano da Faculdade de Ciências da Universidade do Porto. Psicologia: Teoria, Investigação e Prática, 7(2), 321-333.

Guerreiro-Casanova, D. C., \& Polydoro, S. A. J. (2010). Integração ao ensino superior: relações ao longo do primeiro ano de graduação. Psicologia: Ensino \& Formação, 1(2), 85-96.

Horton, R. (2015). The Impact of a Mentoring Relationship on College Adjustment and College-related Stress for Firstyear, First-generation College Students. Tese de doutorado não publicada, Texas Woman's University, Texas.

Igue, E.A., Bariani, I. C. D., \& Milanesi, P. V. B. (2008). Vivência acadêmica e expectativas de universitários ingressantes e concluintes. Psico-USF, 13, 155-164. doi:10.1590/S1413-82712008000200003

Instituto Brasileiro de Geografia e Estatística (2017). Estimativas da população residente no Brasil e unidades da Federação com data de referência em $1^{\circ}$ de julho de 2017. Recuperado de ftp://ftp.ibge.gov.br/Estimativas_de_Populacao/ Estimativas_2017/estimativa_dou_2017.pdf

Ko, M. S. (2015). Ego-resilience and Emotional Intelligence, Stress Coping Strategies \& Nursing Student's Adjustment to College. The Journal of the Korea Contents Association, 15(10), 280-291. doi:10.5392/JKCA.2015.15.10.280

Lazarus, R. S., \& Folkman, S. (1984). Stress, appraisal, and coping. New York, United States: Springer.

Lee, J. W., \& Eo, Y. S. (2013). Effect of clinical stress and stress coping on college adjustment in nursing students. The Korean Journal of Health Service Management, 7(2), 125-134. doi:10.12811/kshsm.2013.7.2.125

Leite, U. R., Tamayo, A., \& Günther, H. (2003). Organização do uso do tempo e valores de universitários. AvaliaçãoPsicológica, 2(1), 57-66.

MacCann, C., Fogarty, G., \& Roberts, R. D. (2012). Strategies for success in vocational education: Time management is more important for part-time than full-time students. Learning and Individual Differences, 22(5), 618-623. doi:10.1016/j.lindif.2011.09.015

Madhyastha, S.; Latha K. S., \& Kamath A. (2014). Stress, coping and gender differences in third year medical students. Journal of Health Management, 16, 315-326. doi:10.1177/0972063414526124

Margis, R., Picon, P., Cosner, A. F., \& Silveira, R. O. (2003). Relação entre estressores, estresse e ansiedade. Revista de Psiquiatria do Rio Grande do Sul, 25(Supl. 1), 65-74. doi:10.1590/S0101-81082003000400008.

Misra, R., \& Mckean, M. (2000). College students' academic stress and its relation to their anxiety, time management, and leisure satisfaction. American Journal of Health Studies, 16(1), 41-51.

Monteiro, C. F., Freitas, J. F., \& Ribeiro, A. A. P. (2007). Estresse no cotidiano dos alunos de enfermagem da UFPI. Escola Anna Nery Revista Enfermagem, 1(11), 66- 72.

Oliveira, C. T., Carlotto, R. C., Vasconcelos, S. J. L., \& Dias, A. C. G. (2014). Adaptação acadêmica e coping em estudantes universitários brasileiros: uma revisão de literatura. Revista Brasileira de Orientação Profissional, 15(2), 177-186.

Oliveira, C. T., \& Dias, A. C. G. (2013). Adaptação acadêmica em estudantes universitários que frequentam ou não o curso desejado. Anais da Jornada Acadêmica Integrada, 28, 1-5.

Oliveira, C. T., \& Dias, A. C. G. (2014). Dificuldades na Trajetória Universitária e Rede de Apoio de Calouros e Formandos. Psico, 45(2), 187-197.

Oliveira, C. T., Carlotto, R. C., Teixeira, M. A. P., \& Dias, A. C. G. (2016). Oficinas de gestão do tempo com estudantes universitários. Psicologia: Ciência e Profissão, 36(1), 224-233. doi:10.1590/1982-3703001482014.

Páramo, M. F., Rodriguéz, M. S., Tinajero, C., Guisande, M. A., Castelo, A. M., Martinez, Z., \& Almeida, L. S. (2010). Relevancia de los sistemas de soporte social y nivel de integración en el marco de la convergencia al espacio europeo de Educación Superior: una propuesta de investigación en la Universidad de Santiago de Compostela. In Actas do Congresso Ibérico Ensino Superior em Mudança: tensões e possibilidades (pp. 441), Braga: Universidade do Minho. 
Pereira, C. A., Miranda, L. C. S., \& Passos, J. P. (2010). O estresse e seus fatores determinantes na concepção dos graduandos de enfermagem. Revista Mineira de Enfermagem, 14(2), 204-209. doi:S1415-27622010000200009

Phun, E. T., \& Santos, C. B. (2010). El consumo de alcohol y el estrés entre estudiantes del segundo año de enfermería. Revista Latino-Am. Enfermagem, 18 (Esp.), 496-503. doi:10.1590/S0104-11692010000700003.

Pimienta, C. C., Cruz, T.C., \& Díaz-Véliz, G. (2016). Ansiedad y fuentes de stress académico em estudiantes de carreras de la salud. Investigación en Educación Médica, 5 (20), 230-237. doi:10.1016/j.riem.2016.03.001

Polo, A., Hernández, J. M., \& Pozo, C. (1996). Evaluación del estrés académico en estudiantes universitarios. Ansiedad y Estrés, 2(2-3), 159-172.

Polydoro, S. A. (2000). O trancamento de matrícula na trajetória acadêmica no universitário: condições de saída e de retorno à instituição. (Tese de Doutorado, Programa de Pós-Graduação em Educação, Universidade Estadual de Campinas).

Ramler, T. R., Tennison, L. R., Lynch, J., \& Murphy, P. (2016). Mindfulness and the college transition: The efficacy of an adapted mindfulness-based stress reduction intervention in fostering adjustment among first-year students. Mindfulness, 7(1), 179-188. doi:10.1007/s12671-015-0398-3

Ross, S. E., Niebling, B. C., \& Heckert, T. M. (1999). Sources of stress among college students. College Student Journal, 33(2), 312-317.

Sadir, M. A., \& Lipp, M. E. N. (2009). As fontes de stress no trabalho. Revista de Psicologia da IMED, 1(1), 114-126. doi:10.18256/2175-5027/psico-imed.v1n1p114-126

Santos, A. F., \& Alves Jr., A. (2007). Estresse e estratégias de enfrentamento em mestrandos de Ciências da Saúde. Psicologia: Reflexão e Crítica, 20(1), 104-113. doi:10.1590/S0102-79722007000100014.

Santos, A. S., Oliveira, C. T., \& Dias, A. C. G. (2015). Características das relações dos universitários e seus pares: implicações na adaptação acadêmica. Psicologia: Teoria e Prática, 17(1), 150-163.

Scopelliti, M., \& Tiberio, L. (2010). Homesickness in university students: the role of multiple place attachment. Environment and behavior, 42(3), 335-350. doi:10.1177/0013916510361872

Soares, A. B., Baldez, M. O. M., \& Mello, T. V. S. (2011). Vivências acadêmicas em estudantes universitários do estado do Rio de Janeiro. Interação em Psicologia, 15(1), 59-69. doi: 10.5380/psi.v15i1.16049

Soares, S. A. (2008). Gestão do tempo e da comunicação. Mediação, 8(7), 20-27.

Recebido: 01/02/17

Aceito: $30 / 09 / 18$

Sobre os autores:

Ana Cristina Garcia Dias é psicóloga, mestre em Psicologia pela Universidade Federal do Rio Grande do Sul (UFRGS), doutora em Psicologia pela Universidade de São Paulo (USP) e professora do Programa de Pós-graduação em Psicologia da Universidade Federal do Rio Grande do Sul (UFRGS).

Rodrigo Carvalho Carlotto é psicólogo e mestre em Psicologia pela Universidade Federal de Santa Maria (UFSM). Atualmente é psicólogo no Instituto Federal Farroupilha - Campus Júlio de Castilhos.

Clarissa Tochetto de Oliveira é psicóloga e mestre em Psicologia pela Universidade Federal de Santa Maria (UFSM). Atualmente, é doutoranda no Programa de Pós-graduação em Psicologia da Universidade Federal do Rio Grande do Sul (UFRGS) e bolsista CNPq.

Marco Antônio Pereira Teixeira é psicólogo é mestre e doutor em Psicologia pela Universidade Federal do Rio Grande do Sul. Professora do Programa de Pós-graduação em Psicologia da Universidade Federal do Rio Grande do Sul (UFRGS). 